\title{
EchoGéo
}

27 | 2014

Structures et armatures urbaines

\section{Ici commence le Brésil ! Géohistoire d'une frontière compliquée}

Federico Ferretti

\section{(2) OpenEdition}

1 Journals

\section{Édition électronique}

URL : https://journals.openedition.org/echogeo/13763

DOI : $10.4000 /$ echogeo. 13763

ISSN : 1963-1197

\section{Éditeur}

Pôle de recherche pour l'organisation et la diffusion de l'information géographique (CNRS UMR 8586)

Référence électronique

Federico Ferretti, « Ici commence le Brésil ! Géohistoire d'une frontière compliquée », EchoGéo [En ligne], 27 | 2014, mis en ligne le 30 mars 2014, consulté le 10 août 2021. URL : http:// journals.openedition.org/echogeo/13763; DOI : https://doi.org/10.4000/echogeo.13763

Ce document a été généré automatiquement le 10 août 2021.

EchoGéo est mis à disposition selon les termes de la licence Creative Commons Attribution - Pas d'Utilisation Commerciale - Pas de Modification 4.0 International (CC BY-NC-ND) 


\title{
Ici commence le Brésil ! Géohistoire d'une frontière compliquée
}

\author{
Federico Ferretti
}

1 Carlo Romani,2013. Aquí começa o Brasil! Histórias das gentes e dos poderes na fronteira do Oiapoque. Rio de Janeiro, Editora Multifoco, 215 p. [Ici commence le Brésil! Histoires des gens et des pouvoirs dans la frontière de l'Oyapock]

2 «L'histoire d'un lieu n'est jamais une histoire unique : elle se présente sous la forme d'un faisceau d'histoires multiples » (Romani, 2013, p. 15). C'est par ces mots que Carlo Romani, historien de l'Université de Rio de Janeiro (UNIRIO), spécialiste de la frontière nord et de l'histoire des mouvements sociaux au Brésil, commence son ouvrage consacré aux histoires de la frontière de l'Oyapock entre le Brésil et la Guyane française. L'idée de la multiplicité de l'histoire d'un lieu prend en compte la nature multidimensionnelle de cette dernière, y compris sa géographicité. L'auteur aborde à la fois l'histoire environnementale et l'histoire humaine d'une région où les frontières continuent à lui sembler une création artificielle par rapport à la circulation humaine traditionnelle.

3 Cet ouvrage renvoie à plusieurs débats contemporains de la géographie. Tout d'abord, il participe au débat sur la nature des frontières, considérées comme objets consubstantiels de la construction nationale (Velasco-Graciet, 2005) et donc, comme l'expose Romani, de l'invention géographique de la nation brésilienne. Par ailleurs, il apporte des éléments de réflexion aux questionnements sur les politiques récemment déployées par la France et le Brésil sur la frontière de l'Oyapock, dans la perspective d'une "intégration sud-américaine" de la Guyane (Granger, 2008) voire d'un « décloisonnement » de l'enclave guyanaise par rapport à son grand voisin brésilien (Boudoux d'Hautefeuille, 2010).

4 Comme l'écrit le géographe français et spécialiste de la Guyane Stéphane Granger dans sa préface, peu de personnes connaissent cette région, et moins encore savent, lorsque cet ouvrage paraît, que « un pont connectant l'Amapá et la Guyane française sur les eaux de l'Oyapock attend son inauguration, prévue en 2014 »(Romani, 2013, p. 7). 
5 L'histoire dressée par Romani commence par le conflit entre deux grammaires culturelles qui sont en même temps des grammaires territoriales. Ce conflit est engendré par la rencontre entre les peuples autochtones et les conquérants européens $\mathrm{du} \mathrm{XVI}^{\mathrm{e}}$ siècle. En mobilisant les outils conceptuels de Deleuze et Guattari, l'auteur affirme que "les peuples de langue tupi pourraient être définis, en des termes occidentaux, comme caractéristiques d'une espèce de nomadisme culturel » (Romani, 2013, p. 19) tandis que les navigateurs espagnols et portugais (et anglais, français et hollandais ensuite), avaient d'abord l'habitude de délimiter les territoires et de les nommer, dans un souci de domination des territoires eux-mêmes. D'où le problème, dans les siècles suivants, de retrouver le fleuve où débarqua en 1513 la flotte de Vicente Yañez Pinzon (ou Pinçon pour les lusophones; 1460-1514), baptisé comme El Rio de Vicente Pinzon, tandis que l'étymologie des définitions d'origine indigène utilisées successivement, Japoc, Yapoc, Wayapoc, etc., n’a jamais été complètement clarifiée, au prix des complications diplomatiques que nous allons évoquer.

6 D'après Romani, «la différence entre les deux nominations souligne d'abord une différence de mentalité et de perspective entre les deux civilisations qui s'affrontèrent ici. Pour l'une, celle des anciens habitants, nommer signifiait associer un lieu à une image, à un fait ou à une action déjà connue et existant dans la nature, et cela à titre de référence. Pour l'autre, celle des conquérants qui arrivèrent et essayèrent de soumettre et de dominer les indigènes, nommer signifiait éterniser quelqu'un dans un lieu (...); baptiser des lieux, renommer des dénominations anciennes fut toujours une manière de conquérir et d'occuper des territoires » (Romani, 2013, p. 26).

7 Cette rencontre tragique devait avoir des conséquences durables dans la géographie de la région ; parmi les auteurs évoqués par Romani on trouve plusieurs géographes, dont le premier est Élisée Reclus (1830-1905). Le géographe anarchiste expliquait, dans sa Nouvelle Géographie universelle, les différentes stratégies de territorialisation des Portugais qui ont effectué des transferts de population, et des Français, en observant que la future Cayenne resta longtemps « un repaire pour boucaniers, aventuriers solitaires ou au service de la royauté " (Romani, 2013, p. 31). Du côté lusitain, une colonisation principalement masculine favorisa la création d'une population métissée, gardant des éléments des cultures et des genres de vie indiens, y compris un rapport au territoire qui ne tenait pas forcement compte des frontières établies en Europe.

8 Un point central du discours de Romani est que pendant toute la période des controverses frontalières entre France et Portugal (et Brésil ensuite), c'est-à-dire entre la fin $\mathrm{du} \mathrm{XVII}$ siècle et le début $\mathrm{du} \mathrm{XX}$, «pour la population résidante, cette dispute était assez indifférente. Portugaise, française, brésilienne n'étaient pas des nationalités reconnues par les Noirs, Créoles, Caboclos et Indiens habitant le territoire (...) ce qui était en jeu était simplement le lieu où l'on pouvait vivre, ou survivre » (Romani, 2013, p. 35).

9 Cependant, l'ouvrage analyse, à travers un méticuleux travail sur des archives françaises et brésiliennes (seules les Archives diplomatiques de Nantes manquent à la liste, ce qui peut être regretté), l'histoire de la dispute territoriale. Si pendant le XVII et le XVIII siècle plusieurs traités, dont les plus célèbres sont ceux d'Utrecht de 1713 , ont tranché de manière plus ou moins vague entre les ambitions des couronnes de France et du Portugal, c'est à partir des guerres napoléoniennes que la Guyane devient un enjeu sensible, notamment par son occupation de la part des Portugais. Pendant la Restauration ce territoire est rendu à la France, sans que toutefois soit résolu le 
problème de l'aire comprise entre l'actuel Oyapock et l'Araguary (illustration 1), arrangée provisoirement par la neutralisation de la région en 1841, obtenue grâce à l'entremise de l'Angleterre qui sollicite cet accord «dans la même période où il était question de neutraliser le litige anglo-brésilien (Burnett, 2000) dans la région de Pirara » (Romani, 2013, p. 43).

\section{Illustration 1 - Carte du Contesté Franco-Brésilien}

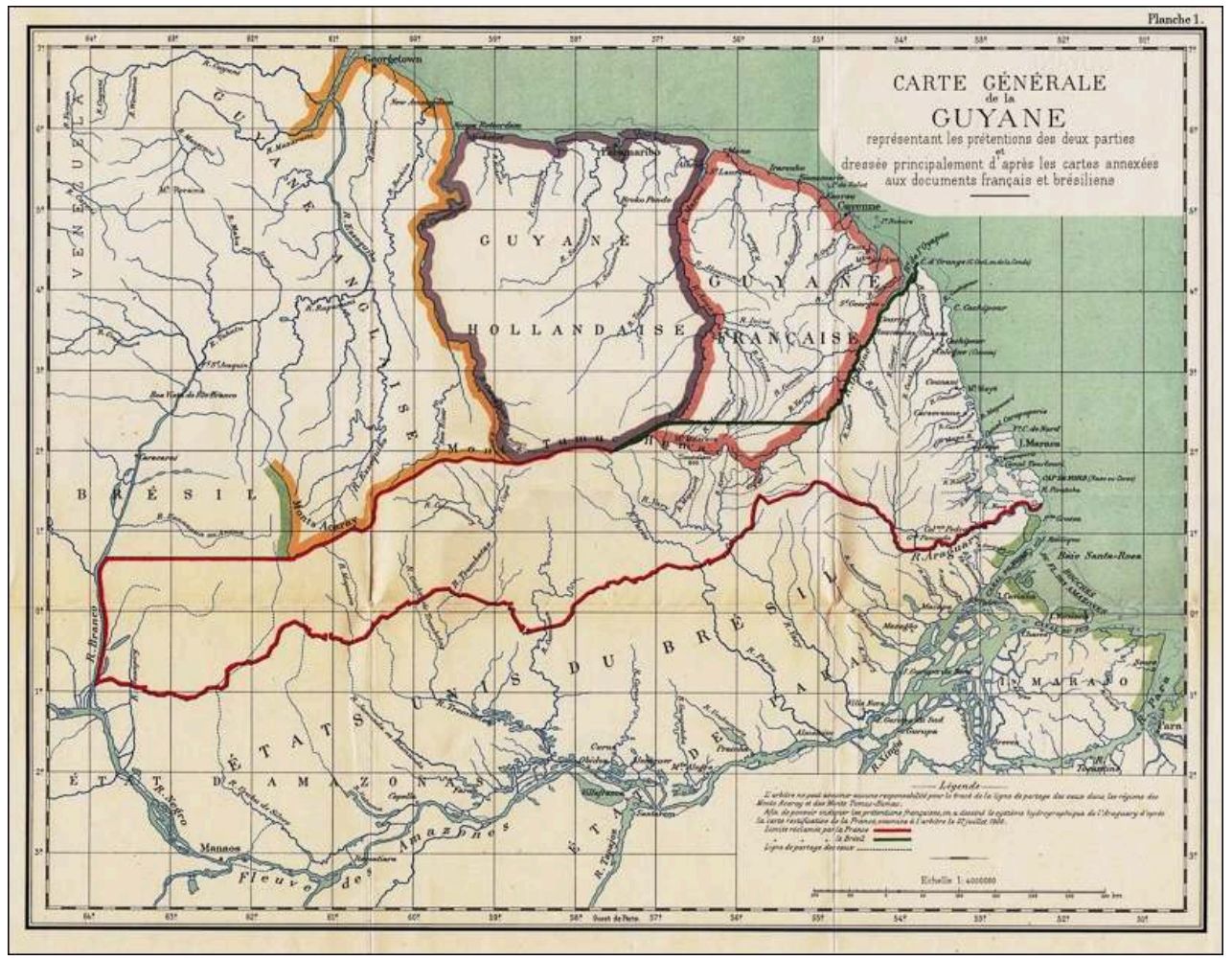

Source : Bibliothèque de Genève, Département de Cartes et Plans, tiroir Amérique du Sud.

Il n'est pas encore question d'établir dans ces territoires des populations européennes, par crainte des contraintes climatiques; dans le secteur français on décide alors d'y établir la colonie pénale de Cayenne.

Ce n'est que dans la dernière décade du siècle que le contentieux territorial fera vraiment rage ; cela, d'après Romani, à cause de l'intérêt renouvelé du Brésil, alors qu'avec la fin de l'Empire et l'avènement de la République en 1889, le capital local déclenche " un véritable impérialisme brésilien » (Romani, 2013, p. 73). Du côté de la France, c'est dans ce même activisme du monde économique et commercial que l'auteur inscrit les explorations de cette période, dont les représentants les plus célèbres furent Jules Crevaux (1847-1882) et Henri Coudreau (1858-1898).

Romani souligne le rôle joué par la géographie dans ces entreprises toujours en équilibre entre science, politique et exploitation commerciale, nouveau modèle dont Coudreau serait l'initiateur. En 1883, «le jeune géographe trouva appui pour une mission d'exploration des richesses territoriales qui devint en même temps une mission politique, dans le sens de l'intérêt national et de la raison d'État, et une entreprise commerciale, inaugurant donc une nouvelle ère d'exploration économique dans le territoire contesté » (Romani, 2013, p. 56). 
13 C'est par l'entremise du capital international qu'on peut expliquer, d'après Romani, certaines expériences éphémères comme la République du Counani (1887-1889), fondée par Jules Gros (1809-1891), qui n'a pas manqué de susciter l'ironie des géographes français, depuis Élisée Reclus jusqu'à Jean-Yves Puyo. Le deuxième citant le premier, il se raconte qu'alors «Paris s'égaya de l'histoire d'un honorable géographe de Vanves soudain transformé en chef d'un État au nom naguère inconnu ${ }^{1}$, et qui s'entoura aussitôt d'une cour, constitua son ministère et fonda un ordre national, l'Etoile de Counani, avec plus de commandeurs, grand-croix, officiers et chevaliers que ne contenait d'habitants la capitale de la république. Mais ce gouvernement dura peu : une année ne s'était pas écoulée que le ministre destituait le président de la nouvelle communauté politique. L'État indépendant de Counani avait disparu » (Reclus, 1894, p. 86, cit. par Puyo, 2011, p. 287). D'après Romani, c'est surtout à cause de la présence de capitaux anglais, intéressés par la probabilité de découvertes de richesses minières dans le territoire contesté, que la France se résout à interdire formellement «toute tentative d'établissement d'un gouvernement libre dans la région, n'acceptant pas la légitimité d'intermédiaires » (Romani, 2013, p. 67).

14 C'est à tous ces aspects "irréguliers » d'une population cosmopolite et migrante que Romani s'intéresse, en analysant par exemple la figure de Trajano, ancien esclave fuyard, collaborateur de Gros et ami de Coudreau, qui, tombé prisonnier des Brésiliens fut considéré comme un traître à la " patrie », tandis que l'auteur le considère comme un exemple de l'humanité apatride qui peuplait le territoire contesté. «Trajano n'était pas brésilien, ni étranger, ni d'aucun lieu : aucune patrie n'a accouché de lui. Il est un non-sens, accepté comme vérité pour l'établissement d'une histoire officielle, que de prétendre la fidélité de quelqu'un à une patrie qui ne l'a jamais voulu libre » (Romani, 2013, p. 88).

15 C'est au milieu de cette humanité que choisit de vivre un personnage controversé et encore peu connu comme Coudreau, dont on sait qu'il n'était pas apprécié par la bourgeoisie créole de la Guyane ; son passage au service du gouvernement brésilien en 1894 fut considéré comme une trahison, comme le confirme son biographe contemporain Sébastien Benoit, qui le définit comme un personnage «un peu anarchiste » (Benoit, 2000, p. 123), rêvant d'une Amazonie indépendante (Romani, 2013, p. 61) et en conflit perpétuel avec les instances officielles.

Ironiquement, de nouveaux documents, ses correspondances avec un «vrai » anarchiste, Reclus, dans lesquelles il se plaint de sa marginalisation et se compare à un "irrégulier ", viennent jeter de la lumière sur ce côté hétérodoxe de Coudreau. « Il paraît que toute la presse opportuniste m'a accusé des plus noirs forfaits : manque de patriotisme, pas d'aptitude à souscrire des pots-de-vin (...), je suis maintenant dans l'état d'esprit où vous étiez en 1871 dans le préau de la prison de Versailles, où les belles donzelles bousculaient du bout de leur ombelle ceux qui paraissaient morts, jouissaient peut-être du sommeil éternel, pour voir comment pourrait être le regard d'un irrégulier abattu $»^{2}$.

17 Ces documents démontrent que la collaboration entre Coudreau et Reclus a joué, bien qu'indirectement, un rôle diplomatique de premier plan, car les documents conservés dans la collection cartographique de Reclus à Genève ont été décisifs pour orienter l'arbitrage de la Confédération helvétique qui a tranché en 1900 sur la question du territoire contesté (Ferretti, 2013). 
18 Selon Romani, c'est à la suite de la découverte de l'or dans l'intérieur des terres et d'incidents comme l'épisode de 1895 , lorsqu'une expédition militaire française atteignit la ville d'Amapá en laissant plusieurs morts sur le terrain, que France et Brésil se décidèrent à chercher une solution définitive par le biais de l'arbitrage suisse. Comme le même auteur le souligne, le rôle de la géographie dans cette controverse fut central : le livre de Coudreau sur la France équinoxiale fut le plus cité dans les mémoires des deux prétendants, l'un écrit par le Baron de Rio $\mathrm{Branco}^{3}$ et l'autre confié à rien moins que Vidal de la Blache (Romani, 2013, p. 59).

19 La question sur laquelle les arbitres devaient s'exprimer a été considérée par Guy Mercier comme un "absurde géographique» (Mercier, 2009), car il s'agissait d'identifier le fleuve de Vicente Pinzon, reconnu comme limite légitime par les traités d'Utrecht, alors que les parties prenantes n'avaient aucune idée de la localisation de ce fleuve parmi les nombreux cours d'eau caractérisant une région très complexe et changeante du point de vue hydrographique. Si le Conseil fédéral tente un travail méticuleux en confiant à William Rosier (géographe genevois ami de Reclus) parmi d'autres une volumineuse étude de la cartographie historique de la région (Ferretti, 2013), ce seraient plutôt des considérations de géographie humaine qui ont décidé de l'arbitrage en faveur du Brésil. Paradoxalement, d'après Romani, les arguments de Vidal de la Blache, très convaincants du point de vue historico-archivistique, " négligèrent la participation humaine dans la question géographique » (Romani, 2013, p. 119). Cela s'explique, selon l'historien brésilien, par un manque de connaissance directe de la région, tandis que l'activisme diplomatique du Baron de Rio Branco fut plus convaincant, parce qu'il démontrait avoir « une relation meilleure avec le savoir local »(Romani, 2013, p. 120).

20 Il faut souligner que Rosier, qui considère comme décisive une carte manuscrite faite par Coudreau pour la Nouvelle Géographie universelle de Reclus (Ferretti, 2013), cite les travaux où ces deux derniers géographes remarquaient le très faible peuplement français dans le territoire contesté. En faisant cela, les arbitres suisses jouent habilement sur la nationalité française de ces deux auteurs, ce qui nous semble décisif, car il démontre que le pouvoir stratégique de la géographie ne s'exprime pas toujours comme simple service à la cause nationale et à l'entreprise coloniale. Ces deux géographes hétérodoxes, Reclus et Coudreau, dont le premier clairement opposé au colonialisme français (Ferretti et Pelletier, 2013) et le second gardant tout à fait une position indépendante (Benoit, 2000), qui poussent leur autonomie jusqu'à aller contre les intérêts de leur nation en se reconnaissant justement dans cet univers humain apatride décrit par Romani, en sont un exemple.

Cela n'est même pas un hasard de la part de Reclus : si le géographe anarchiste n'entre qu'indirectement dans le contentieux franco-brésilien, nous savons par d'autres sources qu'il est consulté systématiquement par la diplomatie brésilienne sur les controverses frontalières de cette époque. Ainsi, le Baron de Rio Branco échange directement avec lui au sujet des frontières contestées avec la Bolivie et l'Argentine (Viana Filho, 1988) ${ }^{4}$ et, en 1903, une mission diplomatique dirigée par un autre " père de la patrie ", Graça Aranha, ${ }^{5}$ rejoint Reclus à Bruxelles pour lui demander sa collaboration en vue d'une nouvelle controverse avec la Guyane anglaise (Castro Azevedo, 2002). D'après la biographie d'Aranha, le géographe anarchiste aurait eu une certaine satisfaction à entraver ainsi l'impérialisme britannique. En l'absence d'un témoignage de l'intéressé sur cet épisode, nous pouvons supposer qu'il n'était pas plus 
enthousiaste à propos de l'impérialisme brésilien, mais il ne faut pas oublier qu'à cette époque l'idée de libération nationale était encore envisagée comme proche de celle de libération sociale et que tout cela relève de rapports complexes entre géographie, anticolonialisme, nationalisme et radicalisme politique qui ont caractérisé cette période. L'exemple du contentieux guyanais apporte des éléments originaux à ce sujet. Les derniers chapitres du livre de Romani sont consacrés aux problèmes que l'État brésilien dut affronter une fois reconnue sa souveraineté nominale sur ce vaste territoire d'environ 270000 kilomètres carrés, correspondant à son " pouvoir de droit et impuissance de fait» (Romani, 2013, p. 119). Après la solution diplomatique du contentieux, « la vie continua son cours naturel dans une région où l'idée d'État n'était qu'un mauvais mirage, se matérialisant uniquement sous la forme d'un poste de douane qui interrompt le libre flux du commerce fluvial » (Romani, 2013, p. 135). L'arrivée du pouvoir politique, d'après Romani, fut tragique sous plusieurs aspects. Au début on se limita à déplacer comme colons des agriculteurs du Ceará, région pauvre du Nord-est brésilien mais de colonisation plus ancienne, ce qui devait aussi «discipliner " et «moraliser » une population hétérogène de métis et migrants, caractérisée "par une profonde circulation culturelle qui nous permet de parler de connexion caraïbeamazonienne » (Romani, 2013, p. 150).

Mais le pire fut, en 1925, l'institution d'une véritable Cayenne brésilienne, le camp de concentration de Clevêlandia où, en raison de la position ultrapériphérique de l'endroit et des accords pour l'extradition en vigueur avec la France, fut déporté un millier de protagonistes de la « révolution pauliste » de 1924. Dans les deux années suivantes, au moins la moitié d'entre eux fut victime d'épidémies, ce qui bouleversa profondément la société locale, au point que personne ne put plus enlever à Clevêlandia " la renommée d'enfer vert, de boucherie humaine» (Romani, 2013, p. 195). Ensuite, cette aire resta sous administration militaire à la fois sous Getúlio Vargas et sous la dictature militaire des années 1960-1970.

Carlo Romani nous livre l'histoire d'un territoire et de son peuple au-delà des histoires diplomatiques et officielles, qu'il maîtrise par ailleurs, tout en soulignant l'importance de la géographie comme science stratégique à plusieurs niveaux. Pour ce qui est des débats géographiques, cités plus haut, sur les frontières en général et sur la frontière de l'Oyapock en particulier, on peut conclure que ce texte renforce l'idée selon laquelle les frontières sont des inventions politiques qui correspondent à une certaine vision du monde, même lorsqu'elles s'appuient sur des objets naturels comme les fleuves. Cependant, l'étude du monde cosmopolite décrit par Romani pourrait encore fournir des éléments utiles pour inspirer les actuelles politiques de "décloisonnement " et "d'intégration régionale » de ce territoire frontalier, à la lumière de son histoire compliquée.

BIBLIOGRAPHIE

Benoit S., 2000. Henri Coudreau (1858-1898). Paris, L'Harmattan, 152 p. 
Boudoux d'Hautefeuille M., 2010. La frontière et ses échelles: les enjeux d'un pont transfrontalier entre la Guyane française et le Brésil. Cybergeo, Revue européenne de géographie, http:// cybergeo.revues.org/23405

Burnett G., 2000. Masters of all they surveyed: exploration, geography, and a British El Dorado. London, The University of Chicago Press, $298 \mathrm{p}$.

Castro Azevedo M. H., 2002. Um senhor modernista: biografia de Graça Aranha. Rio de Janeiro, Academia Brasileira de Letras, 509 p.

Ferretti F., 2013. Le fonds Reclus-Perron et le contesté franco-brésilien de 1900: une carte inédite qui a décidé des frontières du Brésil, Terra Brasilis, 2, http://terrabrasilis.revues.org/766

Ferretti F., Pelletier Ph., 2013. Sciences impériales et discours hétérodoxes : la géographie d’élisée Reclus et le colonialisme français. L'Espace Géographique, 1, p. 1-14.

Granger S., 2008. La Guyane, un territoire Caraïbe en voie de sud-américanisation. EchoGéo [En ligne], 6 | 2008, mis en ligne le 09 juillet 2008. URL : http://echogeo.revues.org/6503 ; DOI : 10.4000/echogeo.6503

Mercier G., 2009. La géographie de Paul Vidal de la Blache face au litige guyanais : la science à l'épreuve de la justice. Annales de Géographie, 667, 3, p. 294-317.

Puyo J.-Y., 2012. Du contesté franco-brésilien à la République de Counani : histoire édifiante et curieuse d'une cryptarchie éphémère. In Dornel L., Guicharnaud-Tollis M., Parsons M., Puyo J.-Y., Ils ont fait les Amériques, Bordeaux, Presses Universitaires de Bordeaux, p. 279-291.

Reclus E., 1894. Nouvelle Géographie Universelle, vol. XIX. Paris, Hachette, 821 p.

Velasco-Graciet H., 2004. Frontières et nation, objets et effets géographiques consubstantiels. In Debarbieux B., Fourny M.-C. L'effet géographique : construction sociale, appréhension cognitive et configuration matérielle des objets géographiques, Grenoble, Maison des sciences de l'homme-Alpes, p. 55-67.

Viana Filho L., 1988. A Vida do Barão de Rio Branco. Rio de Janeiro, José Olimpio, 458 p.

\section{NOTES}

1. Jules Gros était membre de la Société de Géographie de Paris et auto-proclamé président de la République du Counani.

2. Bibliothèque Nationale de France, Département des Manuscrits Occidentaux, Nouvelles acquisitions Françaises 22914, lettre de H. Coudreau à E. Reclus, 18 février 1896, f. 65.

3. José Maria da Silva Paranhos Júnior (1845-1912), Baron de Rio Branco, fut le représentant diplomatique du Brésil auprès de plusieurs pays et ensuite ministre des affaires étrangères. Il joua un rôle déterminant dans la controverse frontalière franco-brésilienne de 1900 et dans plusieurs affaires analogues, comme la définition des frontières avec l'Argentine, la Bolivie et la Guyane britannique. En raison de son énergie et de son efficacité il fut appelé le "Bismarck brésilien". Sa compréhension de l'utilité des sciences géographiques pour la diplomatie et son ouverture intellectuelle le poussèrent à contacter plusieurs géographes et scientifiques européens, dont Reclus, pour avoir leurs expertises sur ces sujets.

4. Ces échanges sont confirmés par des correspondances entre Reclus et Rio Branco, trouvées récemment au Brésil par David Ramirez Palacios, que je remercie pour m'avoir permis de les consulter avant leur publication. 
5. José Pereira de Graça Aranha (1868-1931), écrivain et diplomate brésilien, fut un représentant de la bourgeoisie intellectuelle libérale, très intéressée à la culture européenne, qui se forma pendant les premières années de la République au Brésil (1889). Pendant quelque temps, sympathisant de l'anarchisme, il était très influencé par Reclus et Kropotkine, qu'il n'arrêta pas d'admirer et de citer.

\section{RÉSUMÉS}

Un nouvel ouvrage de l'historien brésilien Carlo Romani vient d'apporter une contribution importante à la construction géo-historique de la frontière entre le Brésil et la Guyane française, ainsi qu'à l'étude de ses enjeux politiques et de son impact sur la société locale. Nous analysons ce travail à la lumière du rôle joué par les géographes français, notamment Henri Coudreau, Élisée Reclus et Paul Vidal de la Blache, qui sont intervenus avec des positions très hétérogènes dans la controverse frontalière de 1897-1900. Nous soulignons finalement l'actualité de ces débats pour les enjeux présents de l'intégration régionale des territoires concernés.

A new work by the Brazilian historian Carlo Romani gives an important contribution to the geohistorical construction of the border between Brazil and French Guiana, including its political importance and its impact on local society. We analyze this work in the light of the role played by French Geographers, namely Henri Coudreau, Élisée Reclus and Paul Vidal de la Blache, who participated, with very heterogeneous positions, in the 1897-1900 border dispute. Finally, we stress the effectiveness of these debates for the present problems of regional integration in the concerned territories.

\section{INDEX}

Mots-clés : Guyane, Oyapock, frontière franco-brésilienne, Henri Coudreau, Élisée Reclus Keywords : Guiana, Oyapock, Franco-Brazilian border, Henri Coudreau, Élisée Reclus

\section{AUTEUR}

\section{FEDERICO FERRETTI}

Federico Ferretti, Federico.Ferretti@unige.ch, docteur en géographie est collaborateur scientifique au Département de géographie et environnement à l'Université de Genève. Il a publié récemment :

- Ferretti F., 2014. Inventing Italy. Geography, Risorgimento and national imagination: the international circulation of geographical knowledge in the 19th century. The Geographical Journal , DOI: 10.1111/geoj.12068, early view: http://onlinelibrary.wiley.com/doi/10.1111/geoj.12068/ abstract

- Ferretti F., 2013. De l'empathie en géographie et d'un réseau de géographes : la Chine vue par Léon Metchnikoff, Élisée Reclus et François Turrettini. Cybergeo, http://cybergeo.revues.org/ 26127 
- Ferretti F., 2013. They have the right to throw us out: Élisée Reclus' Universal Geography.

Antipode, a Radical Journal of Geography, vol. 45, n. 5, p. 1337-1355. DOI http://

onlinelibrary.wiley.com/doi/10.1111/anti.12006/abstract 